\title{
Revisão das pesquisas brasileiras em avaliação psicológica de habilidades e inteligência de condutores
}

\author{
Fábio Henrique Vieira de Cristo e Silva \\ João Carlos Alchieri \\ Universidade Federal do Rio Grande do Norte
}

\begin{abstract}
Resumo
O campo da avaliação psicológica de motoristas é caracterizado por dificuldades e limitações em sua fundamentação e exercício profissional, necessitando de estudos que sistematizem os conhecimentos produzidos e ofereçam sugestões de pesquisas futuras para o seu desenvolvimento. Nesse sentido, o objetivo deste trabalho foi revisar os estudos empíricos brasileiros sobre instrumentos de avaliação psicológica de habilidades e inteligência utilizados nos processos de habilitação para conduzir veículos. Identificaram-se as publicações nestas temáticas no país, seus principais temas e métodos, bem como listaram-se os resultados obtidos. Apenas 15 publicações foram encontradas. Concluiu-se que, em cinqüenta anos, as pesquisas sobre habilidades e inteligência de motoristas trouxeram limitadas contribuições à questão da validade do processo, não sendo constatado um campo de conhecimentos sólidos em relação aos construtos e critérios da avaliação do comportamento.
\end{abstract}

Palavras-chave: avaliação psicológica; habilidades; inteligência; motoristas; psicologia do trânsito

\begin{abstract}
A review of Brazilian research on psychological assessment of drivers 'abilities and intelligence. The field of psychological assessment of drivers is characterized by difficulties and limitations in both its fundamentals and professional practice, calling for studies which systematize its developments. In this sense, the object of this study is to review the Brazilian empirical studies on the instruments for psychological assessment of the abilities and intelligences adopted in the evaluation processes of drivers. Publications dealing with this topic in the country, as well as their main themes and methods were identified, and the results obtained were listed. Only 15 publications were found. It is concluded that, in fifty years, the research on the abilities and intelligences of drivers have brought limited contributions to the issue of validity of the process, not having been found a solid field of knowledge in relation to the constructs and assessment criteria of their behavior.
\end{abstract}

Keywords: psychological assessment; abilities; intelligence; drivers; traffic psychology

$\mathrm{O}$ automóveis têm influenciado decisivamente o desenvolvimento das sociedades, aumentando a capacidade e a rapidez da locomoção das pessoas, comercialização de produtos, conhecimentos, tecnologias e cultura. Todavia, observam-se sérias e negativas conseqüências nas questões ambientais e de saúde pública, com altos custos sociais e emocionais para os envolvidos (Instituto de Pesquisa Econômica Aplicada, Departamento Nacional de Trânsito \& Associação Nacional de Transportes Públicos, 2006; Marín \& Queiroz, 2000). No Brasil, ao final da década de quarenta, já era possível perceber alguns desses aspectos, principalmente os acidentes de trânsito, levando assim as autoridades a desenvolverem e implementarem, na década seguinte, medidas para conter o aumento nos índices de mortos e feridos (Spagnhol, 1985). A obrigatoriedade do processo de avaliação psicológica - outrora denominado exame psicotécnico - para adquirir a carteira de habilitação foi uma dessas medidas, sendo atualmente uma exigência para todo o território nacional (Hoffmann \& Cruz, 2003; Brasil, 2002). Historicamente, portanto, os psicólogos vêm exercendo suas atividades neste processo, coletando e analisando informações para avaliar as habilidades, a inteligência e a personalidade dos futuros condutores, a fim de definirem como aptos ou inaptos, na perspectiva de colaborar para a segurança viária (Campos, 1978a, 1978b; Forbes, 1954; Rothengatter, 1997; Tortosa \& Montoro, 2002; Vieira, Pereira, \& Carvalho, 1953).

A literatura em psicologia do trânsito, tanto nacional quanto internacional, tem questionado se avaliar aspectos psicológicos dos motoristas traz uma efetiva colaboração para a diminuição dos acidentes ou das infrações. Alguns estudos apontam a existência de poucas pesquisas que comprovam a validade 
preditiva dos testes psicológicos em relação aos acidentes de trânsito e a dificuldade em se estabelecer critérios válidos de segurança (Aberg, 2003; Alchieri \& Stroeher, 2002; Groeger, 2003; Risser, 2003; Silva \& Alchieri, 2007). Evidenciam-se, ainda, algumas críticas ao modelo de atuação profissional dos psicólogos, que enfatiza um enfoque observacionalclassificatório, especialmente em vigor no Brasil, cuja raiz remeteria aos primórdios da psicologia científica. Este se limita a avaliar e classificar as respostas dos avaliados, para decidir se eles devem obter ou renovar sua habilitação, em detrimento de um modelo intervencionista, cuja tônica é a intervenção nos processos humanos para tentar modificá-los, considerando os fenômenos observados como determinados pelo momento e pelo ambiente social (Monterde i Bort, 1987; ver também Rothengatter, 2002).

Embora as primeiras aplicações sobre os testes psicológicos no âmbito do tráfego datem da década de 1920 do século passado (Blasco, 1994; Hoffmann \& Cruz, 2003; Tortosa \& Montoro, 2002), a literatura utilizada para embasar esta prática profissional em nosso país configura-se escassa. Somente na década de 1950 , foram publicadas as primeiras reflexões sobre a seleção psicotécnica de motoristas e sua importância na diminuição dos acidentes de trânsito, bem como, os primeiros resultados de exames psicotécnicos e normas para a população brasileira nos diversos testes usados em exames de habilitação, como os testes de Atenção Difusa, Inibição Retroativa, Visão Noturna e Ofuscamento, Volante Dinamógrafo e Psicodiagnóstico Miocinético - PMK (Campos, 1951, 1973; Vieira et al., 1953). Na década de 1980, Spagnhol (1985), ao analisar o desenvolvimento e as perspectivas da psicologia do trânsito no Brasil, evidenciou que após trinta anos do estabelecimento da obrigatoriedade dos exames psicológicos, apenas seis artigos sobre a validade dos exames psicológicos, estatísticas e reflexões teóricas sobre a ocorrência de acidentes tinham sido publicados. Mais atualmente, Alchieri e Stroeher (2002) identificaram diversos problemas no desenvolvimento da psicologia do trânsito brasileira e concluíram que, desde o início da psicotécnica aplicada ao trânsito, os psicólogos ainda não haviam conseguido responder com precisão o que eles observam nos testes que caracteriza uma indicação ou não à habilitação. Em função da diversidade de critérios utilizados pelos psicólogos, poucos estudos com evidências de validade dos instrumentos usados para avaliar condutores podem ser definidos com clareza. Recentemente, Joly, Silva, Nunes e Souza (2007), analisando a produção científica dos congressos brasileiros de avaliação psicológica, identificaram apenas 6 trabalhos em psicologia do trânsito em 2003, 20 trabalhos em 2005 e 10 trabalhos em 2007, configurando em apenas 3,9\% do total de 934 resumos publicados em todas as edições dos eventos.

Revisões de literatura sobre a influência das características de personalidade na condução veicular têm sido realizadas em determinados momentos. Mira (1984) apresentou resultados de pesquisas conduzidas fora do país e realizadas com o PMK em amostras de motoristas pluriacidentados e não acidentados. Mais recentemente, em outra revisão de literatura, Silva e Alchieri (2007) evidenciaram alguns problemas metodológicos nas pesquisas realizadas até então, apontando uma ausência de resultados conclusivos que justifiquem a manutenção da necessidade da avaliação de características da personalidade para adquirir/renovar a habilitação. Revisões sobre outros aspectos do comportamento que também são definidos no processo de habilitação são necessários do ponto de vista teórico e prático para a psicologia, como forma de acompanhar o conjunto de evidências empíricas que sustentam o processo avaliativo, administrado compulsoriamente a milhares de pessoas todos os anos no território nacional. Assim, na perspectiva de sistematizar os conhecimentos produzidos no campo da avaliação psicológica de motoristas, oferecendo um panorama complementar aos construtos anteriormente revisados, especialmente nos aspectos metodológicos, e na perspectiva de oferecer algumas sugestões para ulteriores pesquisas, desenvolveu-se uma revisão dos estudos empíricos sobre características de habilidades e inteligência de condutores de veículos automotores no Brasil.

\section{Método}

Procedeu-se à identificação e análise das publicações relativas aos construtos acima referidos, desde a divulgação dos primeiros artigos sobre os exames psicotécnicos em motoristas na revista Arquivos Brasileiros de Psicotécnica, na década de 1950, até os mais atuais, publicados até 2006, nos diversos periódicos de psicologia. As buscas foram realizadas por meio: (a) da Biblioteca Virtual em Saúde - Psicologia (BVS-Psi), nas bases de dados eletrônicas Index Psi Periódicos Técnico-Científicos (com mais de 160 revistas brasileiras publicadas desde 1949), PEPsic (com 52 periódicos indexados atualmente) e SciELO (com 207 periódicos indexados), a partir das palavras-chaves trânsito, motorista, psicologia do trânsito e avaliação psicológica de motoristas; (b) das próprias referências bibliográficas dos artigos encontrados; e (c) das referências bibliográficas de trabalhos de revisão de literatura em psicologia do trânsito (Alchieri \& Scheffel, 2000; Alchieri \& Stroeher, 2002; Alves, 1999; Dagostin, 2006; Hoffman \& Cruz, 2003; Mira, 1984; Silva \& Alchieri, 2007; Vieira, Amorim, \& Carvalho, 1956). Uma vez identificados, quando disponíveis online, os artigos foram todos acessados, e quando não, foram solicitados às bibliotecas universitárias que tivessem os periódicos indicados em seus acervos, por meio do Serviço Cooperativo de Acesso a Documentos (SCAD - www.bvs-psi.org.br). Foi necessário recorrer a estas estratégias uma vez que a literatura da área indica que os estudos são escassos e dispersos em diversos periódicos, até mesmo alguns fora de circulação. Os artigos encontrados foram lidos e caracterizados quanto aos principais temas estudados, métodos utilizados, participantes, instrumentos e delineamentos de pesquisa.

\section{Resultados}

Encontrou-se um total de quinze trabalhos de natureza empírica (14 artigos e um trabalho completo em anais), dos quais três são da década de 1950, três de 1980, três da década de 1990 e seis são do período entre 2000 a 2006. Nenhum trabalho empírico sobre este conteúdo foi encontrado em décadas anteriores a 1960 e 1970. A seguir são apresentados resumos de cada artigo, contemplando suas principais contribuições, conforme Tabela 1 . 
Tabela 1

Pesquisas empíricas em avaliação psicológica de habilidades e inteligência de condutores

\begin{tabular}{|c|c|c|c|c|}
\hline \multirow{2}{*}{ Autor/Ano } & \multirow{2}{*}{ Tema de investigação } & \multicolumn{3}{|c|}{ Método } \\
\hline & & $N$ & Instrumentos & Delineamentos \\
\hline $\begin{array}{l}\text { Vieira et al. } \\
(1953)\end{array}$ & $\begin{array}{l}\text { Construção de normas de padronização } \\
\text { (tabelas normativas) } \\
\text { Avaliação das características de habilidades } \\
\text { e inteligência de candidatos à renovação da } \\
\text { habilitação e responsáveis por acidentes }\end{array}$ & 1.044 & $\begin{array}{l}\text { Teste de Atenção Difusa, Inibição Retroativa, } \\
\text { Volante Dinamógrafo, Visão Noturna e } \\
\text { Ofuscamento }\end{array}$ & $\begin{array}{l}\text { Descritivo-exploratório } \\
\text { (verificação dos } \\
\text { resultados de um grupo } \\
\text { de participantes nos } \\
\text { instrumentos) }\end{array}$ \\
\hline $\begin{array}{l}\text { Vieira et al. } \\
(1956)\end{array}$ & $\begin{array}{l}\text { Avaliação das características de habilidades } \\
\text { e inteligência de candidatos à renovação da } \\
\text { habilitação e responsáveis por acidentes }\end{array}$ & 4.935 & $\begin{array}{l}\text { Teste de Atenção Difusa, Tacodômetro, Visão } \\
\text { Noturna e Ofuscamento }\end{array}$ & Descritivo-exploratório \\
\hline $\begin{array}{l}\text { Antipoff } \\
(1956)\end{array}$ & $\begin{array}{l}\text { Influência da idade e da emotividade no } \\
\text { exame psicológico }\end{array}$ & 110 & Atenção Difusa de Lahy & Descritivo-exploratório \\
\hline $\begin{array}{l}\text { Andrade } \\
(1983)\end{array}$ & $\begin{array}{l}\text { Estudo sobre o nível de inteligência e a } \\
\text { relação com conhecimentos para dirigir }\end{array}$ & 120 & Teste de Matrizes Progressivas (Raven) & Descritivo-exploratório \\
\hline $\begin{array}{l}\text { Miranda et al. } \\
\text { (1984) }\end{array}$ & Estudo sobre validade e padronização & 535 & $\begin{array}{l}\text { Aptitude Tester for Automobile Drivers } \\
\text { (Simulador TKK) }\end{array}$ & Descritivo-exploratório \\
\hline $\begin{array}{l}\text { Andrade } \\
(1985)\end{array}$ & $\begin{array}{l}\text { Análise e elaboração de modelo para análise } \\
\text { de teste psicológico }\end{array}$ & 120 & $\begin{array}{l}\text { Teste de Inteligência Não-Verbal - Forma C } \\
\text { (INV-C) }\end{array}$ & $\begin{array}{l}\text { Pesquisa fatorial } \\
\text { (classificação de itens } \\
\text { em agrupamentos, } \\
\text { verificando o poder } \\
\text { explicativo destes) }\end{array}$ \\
\hline $\begin{array}{l}\text { Rozestraten } \\
(1990)\end{array}$ & Estudo sobre validade e padronização & 40 & $\begin{array}{l}\text { Teste de Atenção Difusa, Teste de Atenção } \\
\text { Concentrada (AC), Aptidão para Motorista } \\
\text { Militar, Tempo de Reação, Reprodução de } \\
\text { Figuras (Freitas), Disco de Walther, Group } \\
\text { Embedded Figures Test (GEFT), Teste dos } \\
\text { Três Labirintos, Teste de Interesse (Kuder), } \\
\text { Inteligência Não-Verbal (INV-C) }\end{array}$ & $\begin{array}{l}\text { Pesquisa comparativa } \\
\text { (divisão dos participantes } \\
\text { em dois grupos, } \\
\text { comparando-os) }\end{array}$ \\
\hline $\begin{array}{l}\text { Chiança \& } \\
\text { Chiança } \\
\text { (1999a) }\end{array}$ & $\begin{array}{l}\text { Construção de normas de padronização } \\
\text { (tabelas normativas) }\end{array}$ & 1.000 & Teste Não Verbal de Inteligência (R-1) & $\begin{array}{l}\text { Descritivo-exploratório } \\
\text { (verificação dos } \\
\text { resultados de um } \\
\text { grupo de sujeitos nos } \\
\text { instrumentos) }\end{array}$ \\
\hline $\begin{array}{l}\text { Chiança \& } \\
\text { Chiança } \\
\text { (1999b) }\end{array}$ & Construção de normas de padronização & 1.000 & Teste de Atenção Concentrada (AC) & Descritivo-exploratório \\
\hline $\begin{array}{l}\text { Sisto et al. } \\
(2005)\end{array}$ & $\begin{array}{l}\text { Estudo sobre validade e padronização } \\
\text { Nível de inteligência e a relação com } \\
\text { conhecimentos para dirigir }\end{array}$ & 115 & Teste Conciso de Raciocínio (TCR) & $\begin{array}{l}\text { Estudo correlacional (um } \\
\text { grupo de participantes, } \\
\text { observando relação entre } \\
\text { variáveis) }\end{array}$ \\
\hline $\begin{array}{l}\text { Ávila \& } \\
\text { Benczik } \\
(2005)\end{array}$ & $\begin{array}{l}\text { Relação entre acidentes, infrações e } \\
\text { variáveis de habilidade, inteligência e } \\
\text { personalidade }\end{array}$ & 1 & $\begin{array}{l}\text { Teste de Atenção Concentrada (AC) e Teste } \\
\text { Não Verbal de Inteligência (R-1) }\end{array}$ & Estudo de caso único \\
\hline $\begin{array}{l}\text { Montiel et al. } \\
(2006)\end{array}$ & Estudo sobre validade e padronização & 139 & $\begin{array}{l}\text { Teste de Atenção Concentrada Toulouse- } \\
\text { Piéron e Teste de Atenção Concentrada para } \\
\text { Motoristas - Forma A (TACOM-A) }\end{array}$ & $\begin{array}{l}\text { Estudo correlacional (um } \\
\text { grupo de participantes, } \\
\text { observando relação entre } \\
\text { variáveis) }\end{array}$ \\
\hline $\begin{array}{l}\text { Noronha et } \\
\text { al. (2006) }\end{array}$ & Estudo sobre validade e padronização & 212 & $\begin{array}{l}\text { Teste de Atenção Concentrada (AC) e Teste de } \\
\text { Atenção Sustentada (AS) }\end{array}$ & Estudo correlacional \\
\hline $\begin{array}{l}\text { Sisto et al. } \\
(2006)\end{array}$ & Estudo sobre validade e padronização & 65 & $\begin{array}{l}\text { Teste Não Verbal de Inteligência (R-1) e Teste } \\
\text { Conciso de Raciocínio (TCR) }\end{array}$ & Estudo correlacional \\
\hline $\begin{array}{l}\text { Rueda } \\
(2006)\end{array}$ & Estudo sobre validade e padronização & 51 & $\begin{array}{l}\text { Teste Conciso de Raciocínio (TCR) e Teste } \\
\text { Pictórico de Memória (TEPIC-M) }\end{array}$ & Estudo correlacional \\
\hline
\end{tabular}


O primeiro trabalho identificado na literatura foi de Vieira et al. (1953), divulgando os resultados de exames psicológicos e tabelas de diversos testes de aptidão (Atenção Difusa, Inibição Retroativa, Visão Noturna e Ofuscamento, Volante Dinamógrafo). Os autores comentaram críticas sociais feitas ao exame psicotécnico, principalmente, relativas ao prejuízo imposto aos motoristas avaliados como inaptos, do afastamento do meio de vida, ao não recebimento de aposentadoria e o elevado custo dos exames.

Em outro estudo, Vieira et al. (1956) apresentaram resultados de exames de motoristas realizados no antigo Instituto de Seleção e Orientação Profissional (ISOP): teste de Atenção Difusa, Tacodômetro, Visão Noturna e Ofuscamento. A amostra foi aleatória, composta por 4.935 motoristas, divididos em três categorias: candidatos a novas matrículas ou renovação, causadores de acidentes, e motoristas de uma empresa. Os autores expuseram tabelas com a porcentagem de reprovação dos sujeitos por testes de aptidão, que foi de 5,56\% no caso da categoria de causadores de acidentes.

Na mesma década, Antipoff(1956) estudou em 110 pessoas a influência da idade e da emotividade no exame de motorista no teste de Atenção Difusa de Lahy. De acordo com os dados, indivíduos mais novos (entre 18 e 24 anos) se saíram melhor nos exames. Em relação à emotividade, 26\% resistiram à situação do teste e revelaram bastante equilíbrio, diminuindo progressivamente os erros no instrumento.

Posteriormente, na década de 1980, Andrade (1983) realizou investigação sobre a capacidade intelectual em 120 motoristas de transporte coletivo, com o grau de instrução até a 4a série, com o Teste de Matrizes Progressivas de Raven. Os resultados apresentaram uma média de 29,37 acertos no teste, inferior à esperada pela população geral (41,8 acertos), sobre os quais o autor inferiu que os motoristas caracterizavam-se em fase de aquisição do estágio das operações concretas definido por Piaget. A partir dos resultados, o autor sugeriu que treinamentos e campanhas educativas endereçadas a esses motoristas deveriam levar em conta a adequação ao seu nível mental, a fim de que pudessem ser bem compreendidas.

Miranda et al. (1984) desenvolveram estudo de padronização de um simulador para motoristas, o Aptitude Tester for Automobile Drivers (TKK). Este instrumento simulava diversas funções perceptivas, cognitivas e motoras básicas para a condução, como coordenação de movimentos, precisão de reação, distribuição de atenção, estabilidade emocional, visão de cores e interferência da fadiga. A amostra foi composta por 535 brasileiros. Os fatores avaliados foram três: (1) Erros na Aceleração, (2) Erros no Percurso e (3) Acerto na Reação à Cor. Os autores concluíram que, em relação aos fatores 1 e 2, a padronização brasileira seria considerada mais rigorosa que a amostra de referência japonesa, enquanto que no fator 3 o parâmetro seria menos severo.

Em outro artigo, Andrade (1985) estudou 120 motoristas de transporte coletivo, buscando testar um modelo de análise qualitativa da inteligência com base na teoria de Piaget, utilizando o Teste de Inteligência Não-Verbal (INV-C). Foram constatadas inadequações no modelo testado, sendo apresentado um novo modelo e as novas análises constataram melhor adequação da análise qualitativa dos resultados.
Na década seguinte, Rozestraten (1990) realizou estudo para investigar a validade dos instrumentos psicológicos aplicados em motoristas, procurando identificar elementos que diferenciassem um grupo de não-acidentados e outro de pluriacidentados. A amostra foi composta por 20 sujeitos, e foram usados vários instrumentos para medir aptidões e personalidade. No entanto, foram apresentados apenas os resultados das seguintes aptidões: atenção difusa; atenção concentrada (Teste de Cambraia); capacidade visual - acuidade estática, visão cromática e visão noturna - (Teste de Aptidão para Motorista Militar); tempo de reação visual seletiva e auditivo seletivo; coordenação visomotora (Reprodução de Figuras de Freitas); habilidade motora bimanual (Teste de Discos de Walther); estilo perceptivo dependência-independência de campo (Group Embedded Figures Test - GEFT); orientação espacial (Teste dos Três Labirintos); interesses (Kuder); inteligência não-verbal (INV-C) e entrevista. Os resultados não indicaram a existência de um único fator importante que diferenciasse claramente os dois grupos. $\mathrm{O}$ autor concluiu afirmando que, se houvesse alguma diferença, ela deveria ser buscada na personalidade.

Em outro estudo, Chiança e Chiança (1999a) construíram tabela padronizada para o Teste de Atenção Concentrada (AC). A amostra foi de 1000 candidatos à habilitação, com faixa etária entre 18 e 80 anos, com nível de instrução entre o 1 o grau incompleto (5a série) e ensino superior. Assim como no estudo anterior, os dados foram coletados em 1995.

Chiança e Chiança (1999b) desenvolveram ainda um estudo com o Teste Não Verbal de Inteligência (R-1), a fim de construir uma tabela padronizada para avaliar condutores da cidade do Recife-PE. A amostra foi composta também com 1000 examinados, com faixa etária variando entre 18 e 64 anos, com nível de instrução entre o $1^{\circ}$ grau incompleto ( $5^{\text {a }}$ série) e ensino superior. De acordo com os autores, os dados foram coletados em 1995, sendo o estudo publicado somente quatro anos depois.

Outros estudos e mais atuais são encontrados na literatura. Sisto, Bartholomeu e Fernandes (2005), por exemplo, realizaram estudo com o Teste Conciso de Raciocínio (TCR) e uma Prova de Conhecimentos Sobre Trânsito que abordava conhecimentos das normas, habilidades e condutas de risco. Os autores buscaram identificar possíveis relações entre inteligência e conhecimento para conduzir veículos. A amostra foi de 115 estudantes universitários de psicologia e educação física que dirigiam. De acordo com os resultados, os condutores obtiveram bom desempenho no TCR, acertando mais da metade dos itens. Todavia, em relação à prova de conhecimentos, os condutores demonstraram não ter conhecimento das normas de circulação, pouco conhecimento das habilidades necessárias para enfrentar situações adversas no trânsito, com freqüência alta de condutas de risco. Os autores concluíram que existem evidências de validade para o TCR apenas no grupo masculino, sugerindo que, quanto maior a capacidade de raciocínio, maior tendência para o conhecimento das legislações e das habilidades para superar as situações adversas. Não foram observadas associações para o grupo feminino.

Ávila e Benczik (2005) realizaram estudo de caso único de um motorista infrator com 28 anos, solteiro, curso superior incompleto, motorista profissional há 10 anos, que se envolveu 
em três acidentes. Além da entrevista, foram utilizados os testes AC e R-1 e PMK (formato reduzido). No teste de atenção concentrada, o candidato apresentou o nível de atenção dentro da média esperada para sua escolaridade. No nível mental, apresentou bons índices para solucionar os problemas. No PMK, evidenciaram-se alterações no Desvio Secundário Sagital (DSS); traçado disrítmico em todas as folhas e resultado inadequado no DPS; traços disrítmicos, no sentido específico das chamadas Lesões Cerebrais Mínimas. O motorista foi diagnosticado como portador do Transtorno do Déficit de Atenção e Hiperatividade (TDAH) do tipo combinado.

Montiel, Figueiredo, Lustosa e Dias (2006), por exemplo, investigaram a evidência de validade convergente entre o teste de Atenção Concentrada Toulouse-Piéron e o Teste de Atenção Concentrada para Motoristas - Forma A (TACOM-A). Participaram 139 candidatos à obtenção da Carteira Nacional de Habilitação (CNH) de uma clínica credenciada. O TACOM-A relacionou positiva e significativamente apenas com a medida de Rapidez no Toulouse-Piéron. Em relação à medida de Qualidade, os autores não verificaram relações significativas e fortes. Os resultados obtidos foram considerados evidência de validade para a medida de Rapidez do Teste de Atenção Concentrada Toulouse-Piéron.

Noronha, Sisto, Bartholomeu, Lamounier e Rueda (2006) desenvolveram um estudo para identificar evidências de validade para o Teste de Atenção Sustentada (AS) por meio da comparação com o Teste de Atenção Concentrada (AC), em uma amostra foi de 212 candidatos à CNH. De acordo com os resultados, as correlações, embora significativas, não foram fortes. O percentual de comunalidade baixo foi interpretado como sendo um indicador de que a atenção concentrada e sustentada são construtos distintos. Os autores comentaram que os grupos formados em função da sustentação não foram discriminados no Teste de Atenção Concentrada, mas sendo considerados como evidência de validade discriminante para a medida de sustentação do AS. Observando os coeficientes encontrados, os autores inferiram que o termo concentrada, utilizado pelos diferentes testes de atenção, pode estar tendo diferentes conotações.

Sisto, Ferreira e Matos (2006) realizaram estudo com 65 candidatos à $\mathrm{CNH}$ buscando encontrar evidências de validade entre o R-1 e o Teste Conciso de Raciocínio (TCR). Os resultados indicaram aceitável relação entre os instrumentos, sendo que uma parte substancial da variância de ambos os testes foi comum, considerado indicativo de medida de uma quantidade alta de resultados de um mesmo mecanismo psicológico. Os autores consideraram, ainda, que, tanto os níveis mais elementares quanto os mais elevados de raciocínio, não estariam sendo avaliados da mesma forma pelos dois instrumentos, ou então, eles estariam avaliando diferentes mecanismos da inteligência.

Rueda (2006) desenvolveu estudo com 51 candidatos à $\mathrm{CNH}$ para verificar uma possível relação entre memória e inteligência. Utilizaram o Teste Pictórico de Memória (TEPIC-M) e o TCR. Os resultados evidenciaram relações apenas entre um dos três agrupamentos e a pontuação total do TEPIC-M com o TCR. Não foram encontradas diferenças nos resultados dos candidatos em função da categoria de habilitação pretendida (categorias A e B).

\section{Discussão}

Analisando os resultados das publicações empíricas identificadas anteriormente, entre 1953 e 2006, quinze trabalhos foram publicados, em média um a cada três anos e meio. Outras revisões de literatura constataram também esta escassez (por exemplo, Alchieri \& Stroeher, 2002; Dagostin, 2006; Mira, 1984; Silva \& Alchieri, 2007; Spagnhol, 1985), até mesmo de trabalhos apresentados em congressos científicos (Joly et al., 2007). Este aspecto se assemelha bastante aos dados encontrados por Silva e Alchieri (2007), nos estudos sobre personalidade de condutores.

A maioria das publicações - sete - teve como tema principal a validade e padronização dos instrumentos; três desenvolveram estudo para a construção de normas de padronização (tabelas normativas); dois sobre nível de inteligência e sua relação com conhecimentos para conduzir veículos; dois sobre a avaliação das características de habilidades e inteligência de candidatos à renovação da habilitação e responsáveis por acidentes. Outros temas apareceram apenas uma vez, conforme a Tabela 1. Esses estudos, todavia, por serem poucos, não garantem uma medida com fortes evidências de validade tanto para os instrumentos, quanto para a comprovação sistemática das relações entre as variáveis estudadas. Casos cujos participantes têm como escolaridade nível superior incompleto, sem relações demográficas com a população brasileira, ou mesmo, com o interesse de apresentar novos instrumentos de avaliação psicológica sem qualquer evidência de validade, não podem ser tomados como parâmetros científicos relevantes para a justificativa da existência e da utilização do processo avaliativo como atualmente realizado.

A análise dos temas também evidencia que varias questões de ordem prática do cotidiano dos psicólogos permanecem ainda sem resposta, por exemplo: por que os processos relacionados à tomada de informação, processamento de informação, tomada de decisão, comportamento, auto-avaliação do comportamento e traços de personalidade, estabelecidos na resolução do Conselho Nacional de Trânsito no 267, de 15 de fevereiro de 2008, devem ser especialmente avaliados? Existe associação entre essas características e o envolvimento em acidentes/infrações? Como devem se apresentar tais características nos diferentes grupos de condutores (por exemplo, em motoristas profissionais e não profissionais, ou em motoristas com categorias de habilitação distintas) de modo que sejam considerados com desempenho condizente com a condução veicular segura? É oportuno constatar que em nenhum estudo publicado até hoje se estabelecem critérios de avaliação dos candidatos à habilitação e à renovação, ou mesmo aos condutores infratores. A ausência dessas respostas tem favorecido a adoção de critérios aleatórios e particularizados de avaliação do candidato à $\mathrm{CNH}$, conforme evidenciaram Alchieri e Stroeher (2003), ao identificarem mais de 1.000 indicadores apontados pelos psicólogos para classificar um indivíduo apto ou não nos testes de habilidades e 402 indicadores para os testes de inteligência.

Em relação aos métodos de investigação utilizados, e especificamente sobre o número de participantes, a maior quantidade de amostra encontrada foi de 4.935 participantes 
(Vieira et al., 1956), seguido de 1.044 (Vieira et al., 1953), 1.000 (Chiança \& Chiança, 1999a, 1999b), 535 (Miranda et al., 1984) e 212 sujeitos (Noronha et al., 2006). Nas demais investigações, a quantidade foi inferior a 140 participantes. A partir desses dados, observa-se que a busca pela representatividade da população de condutores não tem sido uma meta perseguida nos estudos em avaliação psicológica de condutores. As pesquisas têm sido desenvolvidas sem que critérios demográficos ou outros embasem as análises dos resultados. Não obstante, estudiosos da medida em psicologia recomendam a apresentação de normas de comparação de resultados com considerável número de sujeitos, representando assim diversas características como instrução, idade, sexo e escolaridade, que sejam provenientes de várias regiões do país/estado (Alchieri, Noronha, \& Primi, 2003).

Outro aspecto que chama a atenção é a composição da amostra. Os estudos que objetivaram a construção de tabelas padronizadas basearam-se em amostras de candidatos à habilitação apenas, ou seja, de pessoas não habilitadas. Nesse sentido, será que as características psicológicas e comportamentais avaliadas, especialmente as habilidades, estão sendo bem representadas por estes pequenos grupos normativos? Como avaliar os condutores no ato da renovação da habilitação, com base em uma tabela cujos dados foram obtidos com pessoas não habilitadas? Observa-se que, dos quinze trabalhos, sete tiveram apenas candidatos à habilitação como amostra nos estudos (Chiança \& Chiança, 1999a, 1999b; Miranda et al., 1984; Montiel et al., 2006; Noronha et al., 2006; Rueda, 2006; Sisto et al., 2006). Poucos estudos investigaram motoristas profissionais (Andrade, 1983; 1985; Vieira et al., 1953; 1956), motoristas acidentados e infratores (Ávila \& Benczik, 2005; Rozestraten, 1990; Vieira et al., 1956) e motoristas já habilitados, mas não profissionais (Sisto et al., 2005; Vieira et al., 1956). Noutro artigo, não fica clara a definição da composição amostral (Antipoff, 1956).

Em relação aos instrumentos, foram identificados um total de vinte e dois: dezessete testes de habilidade, quatro de inteligência (R-1, TCR, INV-C, Raven) e um de memória (TEPIC-M). Do total de artigos publicados, quatro realizaram investigações com o AC, três com o R-1, três o TCR, três deles utilizaram o teste de Atenção Difusa de Lahy, dois o INV-C e dois utilizaram o Tacodômetro, Visão Noturna e Ofuscamento. Os demais instrumentos só foram usados apenas uma vez nos estudos. Esses dados sugerem uma diversidade de instrumentos utilizados em pesquisas para avaliar condutores nos construtos de habilidade e inteligência, conforme Alchieri e Stroeher (2003), que identificaram 38 instrumentos ( 29 de habilidade e nove de inteligência). Os testes apresentados não contemplam todos os instrumentos de habilidade e inteligência mais usados no Brasil, como, por exemplo, o TACOM-B, TADIM 1, TADIM 2, TADIS 1, TADIS 2, TRAP-1, conforme identificou o Conselho Federal de Psicologia (2006) e outros (por exemplo, Alchieri \& Stroeher, 2003; Rozestraten, 1984). No caso de alguns dos testes mais usados no país e identificados neste estudo, (R-1, o TACOM-A e o AC), seu uso foi para identificar e diagnosticar características psicológicas dos sujeitos ou auxiliar a validação de outros instrumentos (validade concorrente, por exemplo). Como se pode observar, o foco centra-se nos resultados dos testes, sem que comprovem a validade, tanto em relação ao construto, quanto em relação ao critério (diminuição de acidentes ou infrações de trânsito). Dados semelhantes foram encontrados por Mira (1984) e Silva e Alchieri (2007) nos instrumentos de personalidade. Isto evidencia que, não há nada efetivamente válido para amparar qualquer forma de restrição pautada em uma ação criteriosa, mesmo após deliberações em diversas reuniões e congressos (por exemplo, Rozestraten, 1984). Outro aspecto a ser ressaltado, é que a maioria (64\%) dos instrumentos identificados nas publicações já não é mais usada no processo de habilitação ou ainda não faz parte, até o momento (setembro de 2007), da lista de 95 testes recomendados pelo Conselho Federal de Psicologia (www.pol.org.br). É o caso dos testes: Atenção Difusa de Lahy, Tacodômetro, Visão Noturna e Ofuscamento, Inibição Retroativa, Volante Dinamógrafo, INV-C, Simulador TKK, Teste de Aptidão para Motorista Militar, Reprodução de Figuras de Freitas, Disco de Walther, GEFT, Teste dos Três Labirintos e Kuder.

Quanto ao delineamento, as pesquisas foram categorizadas a partir das sugestões de Wainer (2000). A maioria - sete, dos quinze estudos - desenvolveu pesquisas de caráter descritivoexploratório (verificação dos resultados de um grupo de sujeitos nos instrumentos); outros cinco estudos desenvolveram estudo correlacional (investigando um grupo de participantes e observando relação entre variáveis), conforme a Tabela 1. Os outros delineamentos como: pesquisa comparativa, pesquisa fatorial e estudo de caso único apareceram apenas uma vez cada. Estes dados evidenciam que o foco principal das pesquisas parece estar mais nos resultados dos instrumentos do que na relação entre eles e o comportamento dos motoristas no ambiente do tráfego, conforme apontaram Alchieri e Stroeher (2002). Pouquíssimas investigações utilizaram estudos de caso único ou compararam grupos visando estudar as relações existentes entre aspectos psicológicos medidos e o comportamento no trânsito. Estas constatações são semelhantes ao que ocorre nos estudos de personalidade; a diferença é que nesses últimos, o delineamento mais utilizado tem sido a divisão e comparação entre grupos (Silva \& Alchieri, 2007). Desse modo, os delineamentos de pesquisa parecem restritos e pouco criativos para serem utilizados em uma realidade atual e continuamente mutável. Delineamentos mais sofisticados que comparam resultados dos testes com medidas do comportamento no trânsito, acompanhando os motoristas por um período de tempo, poderiam oferecer mais informações sobre a validade de construto e a validade preditiva dos instrumentos.

\section{Considerações finais}

A realização da avaliação psicológica em condutores e candidatos à carteira de habilitação se constituiu historicamente na principal atividade do psicólogo no contexto do trânsito em uma grande parcela da categoria. Entretanto, apesar dos esforços empreendidos por estudiosos, a investigação em avaliação psicológica de habilidades e inteligência trouxe limitadas contribuições à questão da validade do processo, em relação aos construtos e critérios de avaliação do comportamento. Revisões anteriores de literatura têm registrado isso ao longo do tempo em construtos psicológicos avaliados pelos psicólogos, 
como por exemplo, a personalidade. Atualmente, o panorama permanece praticamente o mesmo no que diz respeito à avaliação das habilidades e da inteligência em candidatos e motoristas. Nos aspectos metodológicos, os delineamentos permanecem repetitivos em relação à aplicação de instrumentos e pouco criativos frente às transformações e complexidades da realidade do trânsito nas atuais metrópoles. O mesmo pode ser dito com relação às amostras pouco representativas da população e compostas na totalidade por escolares, e no uso de instrumentos sem evidência de validade. Com base nos resultados observados, evidencia-se ainda que o trabalho dos psicólogos que realizam avaliação psicológica de motoristas nos Detrans e clínicas credenciadas, salvo algumas poucas exceções, consiste basicamente em aplicar a legislação, que estabelece a obrigatoriedade da avaliação de aspectos psicológicos, sem um conhecimento destes fatores.

Desafortunadamente, em cinco décadas, a pesquisa em avaliação psicológica de trânsito não avançou o suficiente para responder aos questionamentos e anseios básicos da sociedade sobre sua obrigatoriedade e sobre os ganhos efetivos com a segurança no trânsito, que justifiquem o investimento financeiro que é despendido por milhares de cidadãos anualmente no processo. A avaliação psicológica de habilidades e inteligência de condutores tem se configurado, portanto, em uma atividade compulsória e de duvidosa qualidade, do que por uma ação cientificamente embasada, que evidencie empiricamente ganhos com a diminuição de acidentes ou de violações das leis de trânsito. Assim, continua sendo primordial o investimento em pesquisas, conforme vem sendo sinalizado desde as primeiras publicações relativas à psicologia aplicada ao trânsito, sendo necessários estudos sobre a validade preditiva dos instrumentos, com delineamentos que incluam além da quantidade da amostra uma descrição verdadeiramente representativa da população brasileira, a fim de clarificar melhor a relação entre os aspectos psicológicos e o comportamento do motorista.

\section{Agradecimentos}

Os autores agradecem ao $\mathrm{CNPq}$, pela bolsa de mestrado para o primeiro autor.

\section{Referências}

Aberg, L. (2003). Problemas relacionados com a seleção de motoristas e a validade de testes. In R. Risser (Org.), Estudos sobre a avaliação psicológica de motoristas (pp. 25-32). São Paulo: Casa do Psicólogo.

Alchieri, J. C., Noronha, A. P. P., \& Primi, R. (Orgs.). (2003). Guia de referência: testes psicológicos comercializados no Brasil. São Paulo: Casa do Psicólogo.

Alchieri, J. C., \& Scheffel, M. (2000). Indicadores da produção científica brasileira em avaliação psicológica: Resultados da elaboração de uma base de dados dos artigos publicados em periódicos brasileiros de 1930 a 1999. In Pontifica Universidade Católica de Minas Gerais (Org.), VEncontro Mineiro de Avaliação Psicológica e VIII Conferência Internacional de Avaliação Psicológica (pp. 195-197). Belo Horizonte: Autor.

Alchieri, J. C., \& Stroeher, F. (2002). Avaliação psicológica no trânsito: o estado da arte no Brasil sessenta anos depois. In R. M. Cruz, J. C. Alchieri \& J. Sardá (Orgs.), Avaliação e Medidas Psicológicas (pp. 234-345). São Paulo:
Casa do Psicólogo.

Alchieri, J. C., \& Stroeher, F. (2003). Características do processo de avaliação psicológica para condutores de veículos: Um estudo sobre a tomada de decisão dos psicólogos na utilização de testes psicológicos no Brasil. Revista Iberoamericana de Diagnostico y Evaluación Psicológica, 15(1), 107-119.

Alves, I. C. B. (1999). O exame psicotécnico de motoristas. In Instituto Brasileiro de Avaliação e Pesquisa em Psicologia (Org.), VIII Congresso Nacional de Avaliação Psicológica (pp. 216-224). Porto Alegre: Autor.

Andrade, A. S. (1983). Investigação sobre as características intelectuais dos motoristas de transporte coletivo intermunicipal. Psicologia \& Trânsito, 1(1), 52-59.

Andrade, A. S. (1985). Introdução qualitativa da inteligência de motoristas de coletivos baseada na teoria de J. Piaget através do teste INV-C: verificação empírica e revisão do modelo proposto por E. Nick. Psicologia \& Trânsito, 2(1), 31-52.

Antipoff, D. (1956). Contribuição ao estudo das diferenças individuais no teste de atenção difusa. Arquivos Brasileiros de Psicotécnica, 8(2), 49-60.

Ávila, F. A., \& Benczik, E. B. P. (2005). Transtorno de Déficit de AtençãoHiperatividade - TDAH: um estudo do exame psicológico de um motorista infrator. Revista ABRAMET, 22(45), 32-39.

Blasco, R. D. (1994). Psychology and road safety. Applied Psychology: An International Review, 43(2), 313-322.

Brasil (2002). Código de trânsito brasileiro. Brasília: Senado Federal.

Campos, F. (1951). Seleção psicotécnica de motoristas. Arquivos Brasileiros de Psicotécnica, 3(3), 7-26.

Campos, F. (1973). A Divisão de Seleção. Arquivos Brasileiros de Psicologia Aplicada, 25(1), 17-49.

Campos, F. (1978a). O fator humano e os acidentes de trânsito (Primeira parte: Visão geral). Arquivos Brasileiros de Psicologia Aplicada, 30(3), 3-24.

Campos, F. (1978b). O fator humano e os acidentes de trânsito (Segunda parte: A seleção). Arquivos Brasileiros de Psicologia Aplicada, 30(4), 99-117.

Chiança, M. M. R. L. C., \& Chiança, N. A. A. (1999a). Teste de atenção concentrada. Dados coletados dos candidatos à motorista em 1995 - Recife/ PE. PSIC - Revista de Psicologia da Vetor Editora, 1(1), 39-43.

Chiança, M. M. R. L. C., \& Chiança, N. A. A. (1999b). Teste não-verbal de inteligência-R-1. PSIC - Revista de Psicologia da Vetor Editora, 1(1), 45-49.

Conselho Federal de Psicologia. (2006). Pesquisa exploratória sobre o processo de avaliação psicológica para a obtenção da carteira nacional de habilitação. Brasília: Autor.

Dagostin, C. G. (2006). Características do processo de trabalho dos psicólogos peritos examinadores de trânsito na avaliação das condições psicológicas para dirigir. Dissertação de mestrado não-publicada, Universidade Federal de Santa Catarina, Florianópolis.

Forbes, T. W. (1954). Contributions by psychologists to the reduction of highway traffic accidents in the United States of America. Applied Psychology: An International Review, 3(1), 43-58.

Groeger, J. A. (2003). Testes psicológicos podem predizer habilidade para dirigir? In R. Risser (Org.), Estudos sobre a avaliação psicológica de motoristas (pp. 79-95). São Paulo: Casa do Psicólogo.

Hoffman, M. H., \& Cruz, R. M. (2003). Síntese histórica da psicologia do trânsito no Brasil. In M. H. Hoffman, R. M. Cruz, \& J. C. Alchieri (Orgs.), Comportamento humano no trânsito (pp. 15-29). São Paulo: Casa do Psicólogo.

Instituto de Pesquisa Econômica Aplicada, Departamento Nacional de Trânsito, \& Associação Nacional de Transportes Públicos. (2006). Impactos sociais e econômicos dos acidentes de trânsito nas rodovias brasileiras. Brasília: Autores.

Joly, M. C. R. A., Silva, M. C. R., Nunes, M. F. O., \& Souza, M. S. (2007). Análise da produção científica em painéis dos congressos brasileiros de avaliação psicológica. Avaliação Psicológica, 6(2), 239-252. 
Marín, L., \& Queiroz, M. S. (2000). A atualidade dos acidentes de trânsito na era da velocidade: uma visão geral. Cadernos de Saúde Pública, 16(1), 7-21.

Mira, A. M. G. (1984). Resultados das pesquisas realizadas com o PMK em motoristas pluriacidentados e não ou pauciacidentados. Psicologia \& Trânsito, 1(2), 23-32.

Miranda, A. A. P. X., Barbosa, R. J., Lepesqueur, A. C. C., Rodrigues, M., Ribas, D. S., Oliveira, V. L. G., et al. (1984). Padronização de um teste de simulador para motoristas em uma amostra brasileira. Psicologia \& Trânsito, 1(2), 43-52.

Monterde i Bort, H. (1987). La evaluación psicológica por medio del ordenador: validez de constructo y fiabilidad de las actuales pruebas psicológicas para conductores basadas en ordenador. Revista de Psicología del Trabajo y de las Organizaciones, 3(6), 29-47.

Montiel, J. M., Figueiredo, E. R. M., Lustosa, D. B. S., \& Dias, N. M. (2006). Evidência de validade para o teste de atenção concentrada Toulouse-Piéron no contexto de trânsito. Psicologia: Pesquisa \& Trânsito, 2(1), 19-27.

Noronha, A. P. P., Sisto, F. F., Bartholomeu, D., Lamounier, R., \& Rueda, F. J. M. (2006). Atenção sustentada e concentrada: construtos semelhantes? Psicologia: Pesquisa \& Trânsito, 2(1), 29-36.

Risser, R. (2003). Validade dos instrumentos e das atividades do Diagnóstico e Seleção de Motoristas (DSM). In R. Risser (Org.), Estudos sobre a avaliação psicológica de motoristas (pp. 13-19). São Paulo: Casa do Psicólogo.

Rothengatter, T. (1997). Psychological aspects of road user behavior. Applied Psychology: An International Review, 46(3), 223-234.

Rothengatter, T. (2002). Driver's illusions - no more risk. Transportation Research Part F, 5, 249-258.

Rozestraten, R. J. A. (1984). Relatório - II Congresso Brasileiro de Psicologia do Trânsito, de 18 a 21 de agosto de 1983. Psicologia \& Trânsito, 1(2), $15-21$.

Rozestraten, R. J.A. (1990). O uso e a validade dos testes aplicados aos motoristas - dados de uma pesquisa. In Sociedade Brasileira de Psicologia (Org.), $20 \underline{a}$ Reunião Anual de Psicologia (pp. 471-476). Ribeirão Preto: Autor.

Rueda, F. J. M. (2006). Memória e inteligência em avaliação psicológica pericial. PSIC - Revista de Psicologia da Vetor Editora, 7(1), 59-68.

Silva, F. H. V. C., \& Alchieri, J. C. (2007). Avaliação psicológica da personalidade de condutores: uma revisão de literatura. Psico-USF, 12(2), 189-196.

Sisto, F. F., Bartholomeu, D., \& Fernandes, D. C. (2005). Inteligência e conhecimento para conduzir veículos automotores. Psicologia: Pesquisa \& Trânsito, 1(1), 53-62.

Sisto, F. F., Ferreira, A., \& Matos, M. P. B. (2006). TCR e R1: duas medidas do fator G. PSIC - Revista de Psicologia da Vetor Editora, 7(1), 69-77.

Spagnhol, J. M. (1985). A psicologia do trânsito no Brasil: desenvolvimento e perspectivas. Psicologia \& Trânsito, 2(2), 7-10.

Tortosa, F., \& Montoro, L. (2002). La psicología aplicada a la selección de conductores. Cien años salvando vidas. Psicothema, 14(4), 714-725.

Vieira, M. V. M., Amorim, J. A., \& Carvalho, A. V. (1956). O psicodiagnóstico miocinético na seleção de motoristas. Arquivos Brasileiros de Psicotécnica, $8(1), 53-65$.

Vieira, M. V. M., Pereira, A. O., \& Carvalho, A. V. (1953). O exame psicotécnico de motoristas no Distrito Federal. Arquivos Brasileiros de Psicotécnica, 5(4), 41-50.

Wainer, R. (2000). A pesquisa quantitativa em psicologia: delineamentos possíveis e a questão da amostragem. In H. Scarparo (Org.), Psicologia e pesquisa: Perspectivas metodológicas (pp. 17-34). Porto Alegre: Sulina.

Fábio Henrique Vieira de Cristo e Silva, graduado e mestrando em Psicologia pela Universidade Federal do Rio Grande do Norte, é coordenador da Comissão de Psicologia do Trânsito no Conselho Regional de Psicologia/CRP-17. Endereço para correspondência: Av. Rui Barbosa, 1122, Apto. 801-B, Lagoa Nova; Natal, RN; CEP: 59.056-300. Tel.: (84) 3221-2527, 8832-6661. E-mail: fabiodecristo@gmail.com João Carlos Alchieri, doutor em Psicologia do Desenvolvimento pela Universidade Federal do Rio Grande do Sul, é professor no Programa de Pós-graduação em Psicologia da Universidade Federal do Rio Grande do Norte.E-mail: jcalchieri@gmail.com 